\title{
Visualization Support for Multi-criteria Decision Making in Geographic Information Retrieval
}

\author{
Chandan Kumar ${ }^{1}$, Wilko Heuten ${ }^{2}$, and Susanne Boll ${ }^{1}$ \\ 1 University of Oldenburg, Oldenburg, Germany \\ \{chandan.kumar, susanne.boll\}@uni-oldenburg.de \\ 2 OFFIS - Institute for Information Technology, Oldenburg, Germany \\ wilko.heuten@offis.de
}

\begin{abstract}
The goal of geographic information retrieval (GIR) is to provide information about geo-entities to end-users and assist their spatial decision making. In the current means of GIR interfaces, users could easily visualize the geo-entities of interest on a map interface via sequential querying or browsing of individual categories. However, there are several decision making scenarios when the user needs to explore and investigate the geospatial database with multiple criteria of interests, which is not well supported by the sequential querying or browsing functionality of current GIR interfaces. There is a need for more sophisticated visual interfaces to enable end-users in discovering knowledge hidden in multidimensional geospatial databases. In this paper we discuss some of the $\mathrm{HCI}$ issues in realizing such multi-criteria decision making scenario based on the user requirement analysis. To tackle the human centered aspects we propose different heatmap based interfaces to support multi-criteria visualizations in GIR, i.e., to facilitate the knowledge based exploration of geospatial databases with less information overload.
\end{abstract}

Keywords: Local Search, Geographic Information Retrieval, Geovisualization, User Interfaces, User-Centered Design, Heatmaps, Grids, Voronoi diagram.

\section{Introduction}

Geo-related information is one of the basic needs of citizens to understand the local infrastructure and to satisfy their spatial information requirements [1. Spatial databases of georeferenced entities, documents and Web pages are a huge information source for geographic locations and entities. A GIR system supports end-users to search into these spatial databases [11, its goal is to process the information request of end-users and provide the results to satisfy their spatial information need. The current GIR interfaces are able to serve comparably simple requests, e.g., a search for "Restaurant in New York" in Google Map\$1 will return all restaurants in the downtown New York. However,

${ }^{1}$ http://www.maps.google.com

A. Cuzzocrea et al. (Eds.): CD-ARES 2013, LNCS 8127, pp. 363-375, 2013.

(C) IFIP International Federation for Information Processing 2013 
in the current information age, the spatial information need of an end-user is often much more complex than such simple, aforementioned queries.

In several scenarios of spatial decision making users look for multiple criteria of interest simultaneously, which is not very convenient with the sequential querying and categorization approach of current GIR interfaces, e.g., somebody who has to move to a new city and is looking for a new living place. In this example the user will most likely come up with a variety of different criteria of geo-entities that he/she would like to visualize it together, e.g., availability of shopping facilities, medical facilities, and a good connection to public transport. Existing GIR systems fail to satisfy such complex information needs of end-users, even though the associated geospatial databases contain all these multi-dimensional information. The need is to support geographic retrieval with appropriate visualization methods that could assist users to explore the geospatial databases more effectively to discover the hidden spatial knowledge.

In this paper we argue the need of visualization methods for GIR to assist the complex decision making task of end-users. We conducted a user study to understand the requirements of end-users for GIR decision support systems, where we presented some spatial decision making scenarios such as moving to a new region or city. The insights of our study motivated us to design different interfaces which can support users in visualizing multiple criteria on the map with less information overload. We primarily focus on the heatmap visualizations (which have been simple yet effective phenomena in visualization research [16,6] for the information representation with less overload), and proposed various GIR interfaces using aggregated computations of geo-entities with grid-based and voronoi divisions. The proposed interfaces operate with the effective visualizations to enhance the capability of geospatial database in providing useful knowledge about spatial regions, and assist end-users decision making task.

The rest of paper is organized as follows: First, we give an overview of the multi-criteria local search problem and discuss the related applications and research approaches in Section 2. Then in Section 3 we describe our user study which was conducted to characterize multi-criteria search over various scenarios of exploring urban regions. We propose our heatmap based visual interface for multi-criteria local search in Section 4. In this section we describe how grid-based and voronoi- divisions were employed to present the aggregated spatial information. Finally, Section 5 concludes the paper by discussing the contribution of the paper and directions for future work.

\section{Background and Related Work}

Geographic information retrieval or local search provides the information about geo-entities to end-users 111]. These geo-entities usually belong to a criteria/category or set of categories (shopping, education, sport, etc.). Several location-based services and yellowpages follow the categorical structure for the overview of geo-entities. In the current geo applications, users could view the geo-entities which belong to a particular category on the map, but realization of 
multiple categories is usually not supported. So end-users are expected to accomplish decision making task through sequential querying/browsing of categories which could be extremely complex and time consuming task. There are a few commercial application: 23 which provide the end-user interface of few selected categories for a very specific task like hotel or apartment search. In comparison we focus on the generic local search for end-users, and to support their decision making through visualization of multiple categories on a map interface.

In the research community, multi-criteria spatial problems have been approached from the computation perspective [18,22] which is focused on distance and density estimation of locations with respect to different criteria. In comparison, we focus on visualization methods, i.e., how end-users perceive and analyze multiple criteria on local search interface for spatial decision making. Some geovisualization approaches [57] explore the problem of multi-criteria analysis, but they usually target specific domains like healthcare and support the decision analysis of a focused group of experts. Rinner and Heppleston 21] proposed geospatial multi-criteria evaluation for home buyers where decision criteria were based on: location, proximity, and direction. Even though their task has been guided by a similar scenario of spatial decision making like discussed in our work, the study was conducted for a focused group of real-estate agents and the contribution was more on the computation issues rather than the HCI and visualization aspects. In general geospatial decision making has been one of the main challenges and application of visual analytics [10]. For geo-related content visual analytics is prominently being used. There have been many visual analytical models and tools developed to support critical business decision making processes, but assisting lay users in their decision making is still a major research challenge.

In this paper we focus on the analysis of GIR visual interfaces with endusers. The research problem of user-centered analysis have been well investigated in visualization and human-computer interaction research[191217, also in the spatial context of geovisualization[1320]. Even though we follow the general guidelines from the aforementioned work, we particularly focus on the usability and end-user satisfaction of a heatmap based visualizations. It is common to use heatmaps, grid structure, and voronoi divisions, in many visualization approaches, but their impacts on the GIR interfaces to support user's multi-criteria decision making have not been explored before.

In this work we particularly augment the interfaces of our existing GIR system whose goal is to provide the access of geo-entities through spatial Web pages. Figure 1 shows the end-user interface of system which is very similar to the current local search services, i.e., markers on the map to show the geo-entities, and an associated ordered list of results. The associated geospatial database consist a huge collection of georeferenced Web pages through a focused Web crawler [3]. Identification and verification of the addresses are done against the

\footnotetext{
2 http://www.hipmunk.com/hotels/

3 http://www.walksore.com/apartments/
} 


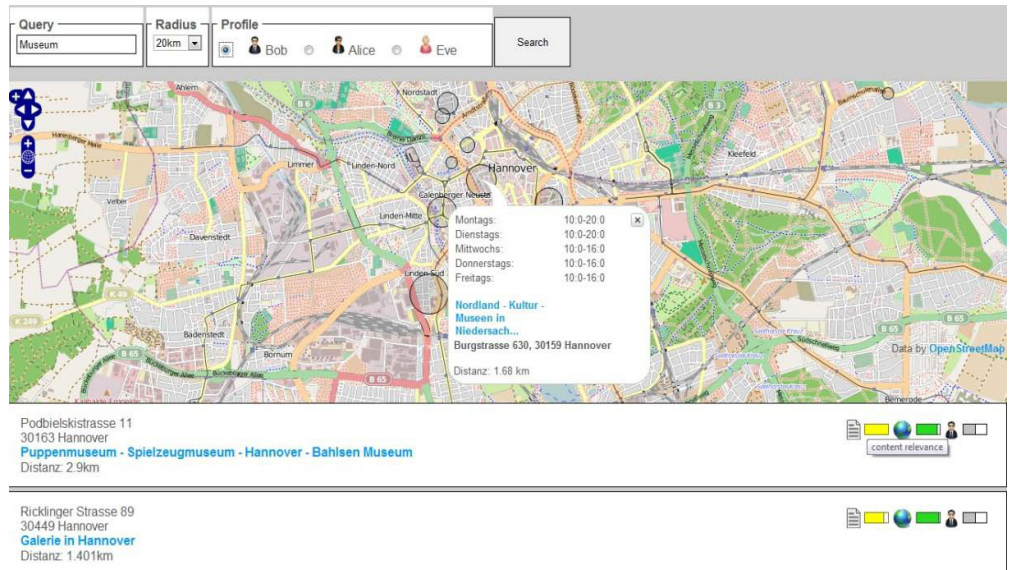

Fig. 1. A screenshot of our early GIR interface

street name database, which we fetched from OpenStreetMan for the major cities of Germany. The system is primarily focused on relevance algorithms and computational ranking solutions [112] to process user requests and listing result documents in a ranked order.

\section{User Requirement Analysis in Complex Spatial Decision Scenarios}

The role of end-user is of prime importance in the successful design of Web interfaces, so the usability and HCI aspects need to be considered 917. We follow the generic guidelines of user-centered design process to understand the context of use and to gather requirements. We conducted a semi-structured interview, where we asked people about experiences with existing systems, gathered insights, desires and ideas for improvements regarding GIR decision making tasks. Ten users participated in our study. The participants were aged between 33 (mean) and 5.6 (standard deviation). We asked the participants to think about how it feels to move to a different city. We thereby hoped that the participants can provide us with more thoughtful, inspiring information. Initially, we asked each participant to name the important criteria and aspects if they would like to move to a different city, or to open up a business in a city, or to compare different cities. Then we asked each participant on how an ideal user interface for these scenarios could look like. Thereby, also non-stationary devices could be considered. Participants were allowed to draw sketches with pen and paper. We then asked them to reflect on how they use existing tools, like Google Maps, the GIR interface (Figure 1), for these scenarios and if they fulfill their needs.

4 http://www .openstreetmap.org/ 


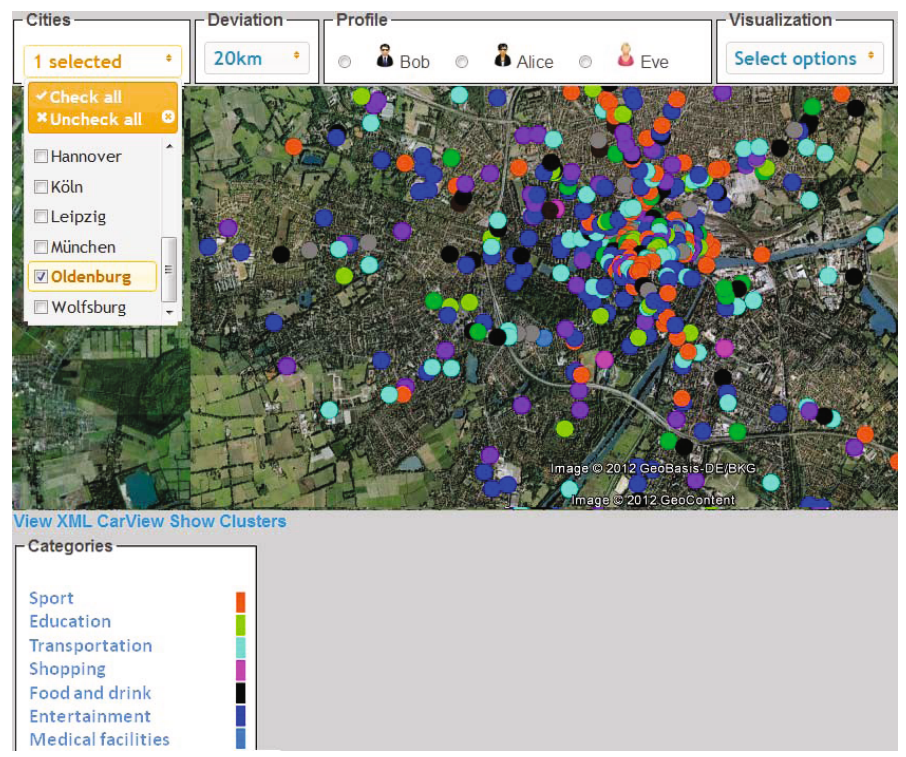

Fig. 2. Marker-based interface for multi-criteria exploration

Ultimately, we confronted the participants with an early functional prototype of our GIR interface for multi-criteria search (Figure 2) and asked them to share their thoughts by thinking aloud. The prototype is the easy adaption of the existing GIR interface to overcome the limitation of sequential querying and browsing. It supports multi-criteria selection through the colored association of markers on map. Each marker (circle) represents a geo-entity and follows the color scheme of its respected category.

We obtained the general perception that the user's requirements could be very diverse and dynamic. All study participants had their very own criteria in mind when discussing about the scenario, some criteria were contradictory, e.g., one participant wants to live as close as possible to work, while another participant wants to live as far away from work as possible to have a clear separation. Most of the participants stated that existing tools and services are not sufficient to fulfill these tasks in a simple and easy way. The current means of interfaces support only the sequential querying and searching of geo-entites, so the exploration with respect to multiple criteria of interests becomes an uneasy task. Few participants mentioned that they could achieve the task via several sequential queries. Moreover, they supported our point of view that this decision making process is very complex and the way to merge information in mind is demanding. Simplification in the information presentation, categorical and aggregated overview, easy and adaptive interaction were the main wishes of the users, we discuss these observations more specifically in the following. 
Simplification of Information Presentation. Most of the users wished for easy and simple visualizations while being asked about their need in realizing an ideal geospatial decision support system. Participants were concerned from the overload of information from existing interfaces and the complexity of representation. The geospatial databases contain multivariate and complex attributes so the need is to summarize this huge information via easy visualizations. In order to support simple visualization, advance interaction methods could be useful to provide exploration capabilities to end user, i.e., to reduce the information loss.

Visualization at Category Level Is Important. We noticed that users tend to visualize the geo attribute at low granularity. While being asked to list the important geo attributes for relocation, users mention the geo-relevance parameters with a categorical overview, e.g. shopping, sports, education etc. The usual geo-data available to search systems are at the high granularity with real physical addresses of local entities. So the need is to represent the geovisualization of spatial database with low level category based details to simplify the initial visualization for end users.

Aggregation of Information and Knowledge-Based Support. In the scenarios such as geovisualization, integration of geo-spatial knowledge, learning of geo-spatially related entities in the search and visualization is required. There is a greater need of visual analysis tools to represent and reason with human knowledge and formalize the intersection between interaction and knowledge construction. From the user study we got the impression that the user is very willing to support these knowledge-based interfaces. In many of the drawn sketches we identified principles to assist such interfaces. Most prominently, the users wanted to set their general priorities and interests on some scales. These could serve as a starting point for building up more detailed knowledge-based interfaces. As open challenge we see how the user's set preferences and intelligent interface support work together, in particular since the user's interests might change over time or for each session.

Adaptive Interaction Techniques. Interfaces of spatial data usually demands interaction patterns like zooming, panning, rotating or selecting. The challenge is to provide users the scope to utilize these techniques to navigate the map and, thus, the geographic search results. The user should be able to mark areas, move to other areas, and manipulates the retrieved attributes till he/she reaches a deep understanding of the data and potentially gathers new knowledge. From the user study we got strong support that interaction techniques beyond traditional WIMP (windows, icons, menus, pointer) interfaces should be considered. We learned that the context is often completely different between users. It might be even different in various situations of the same user. E.g., the user's individual interaction flow might be different to the flow when the interests of a whole family are considered. These different profiles are very hard to infer from a 


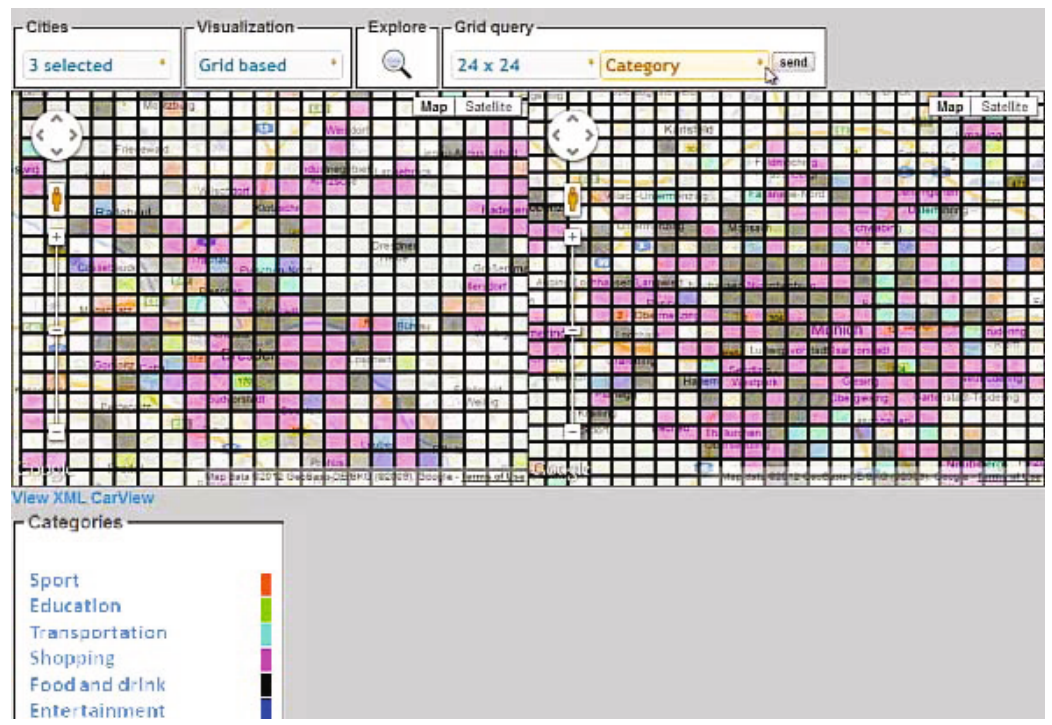

Fig. 3. Grid-based heatmap visualization for spatial exploration

technical point of view. However, the participants appreciated the different user profiles of the shown prototype (Figure 2). Thus, this is a significant challenge that needs to be addressed.

\section{Visualizations to Support Multi-criteria Decision Making in GIR}

Based on the gathered insights we are able to make some fundamental design decisions. We know that people will approach a spatial decision making task, like in the relocation scenario, with a map-based tool. Consequently, we decide that the main part of the user interface should be a map. This map should exactly behave like traditional map-based systems to match the user expectations regarding common interactions like zooming or panning. The user interface should use a simple language, should be easy to understand, should be clearly structured and further should avoid information overload. A trade-off was deemed optimal for the criteria selection process. The users should be allowed to adapt the criteria to their very individual needs. The user preferred criteria gathered in the requirement analysis guided our formulation of the list of category.

Our primary goal is to provide an appropriate visualization of the spatial data for GIR users. In visualization research, there have been many advance multivariate, high dimensional visualizations for analysts and decision makers which could be hard to interpret by lay users. Here we imply the heatmap based information presentation phenomena in the GIR interface, which is easily understandable and less prone to information overload. Heatmap visualization has been applied in various domains to represent spatial distributions and relevance [6]. 


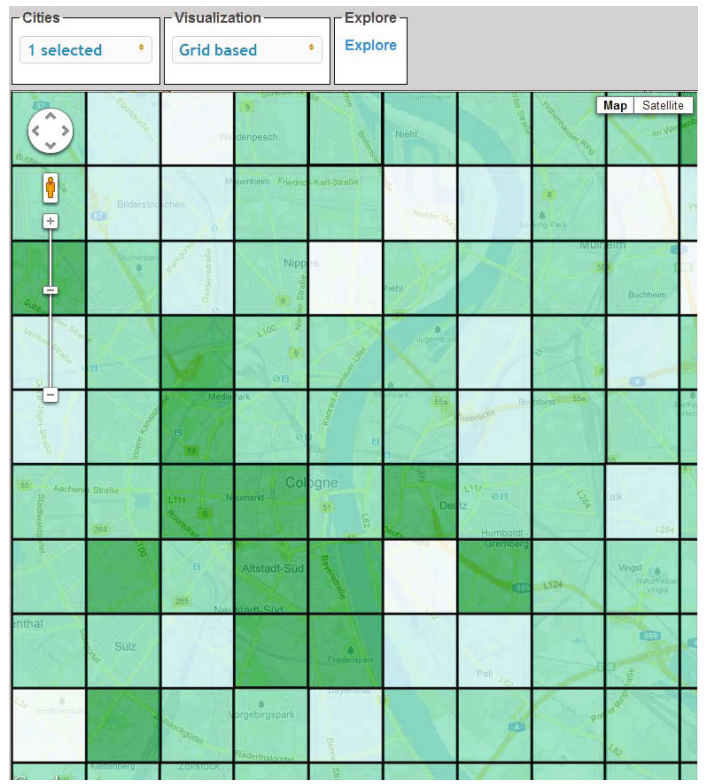

Fig. 4. Ranked grid-based visualization

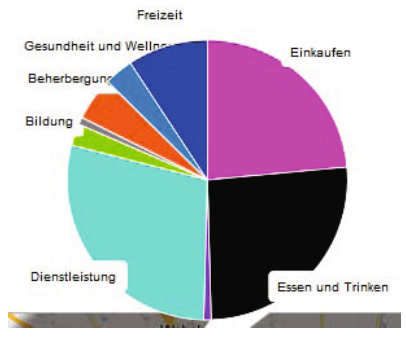

Fig. 5. Pie charts for user interaction

\subsection{Grid-Based Heatmap Visualization for Spatial Exploration}

For the aggregational view of geo-entities we discretize the map area using a grid raster [16, since we didn't intend to restrict user exploration to be constrained by administrative boundaries. We adapted the heatmap visualization for the distribution of categories across user-defined grid-based division of maps. Each grid cell conveys the categorical information. The color of each grid cell represents the category of largest allocation of geo-entities in the cell area. Figure 3 shows the grid-based visualization for two user selected cities with respect to 6 selected categories of interest. We can perceive that the visualization gives a 


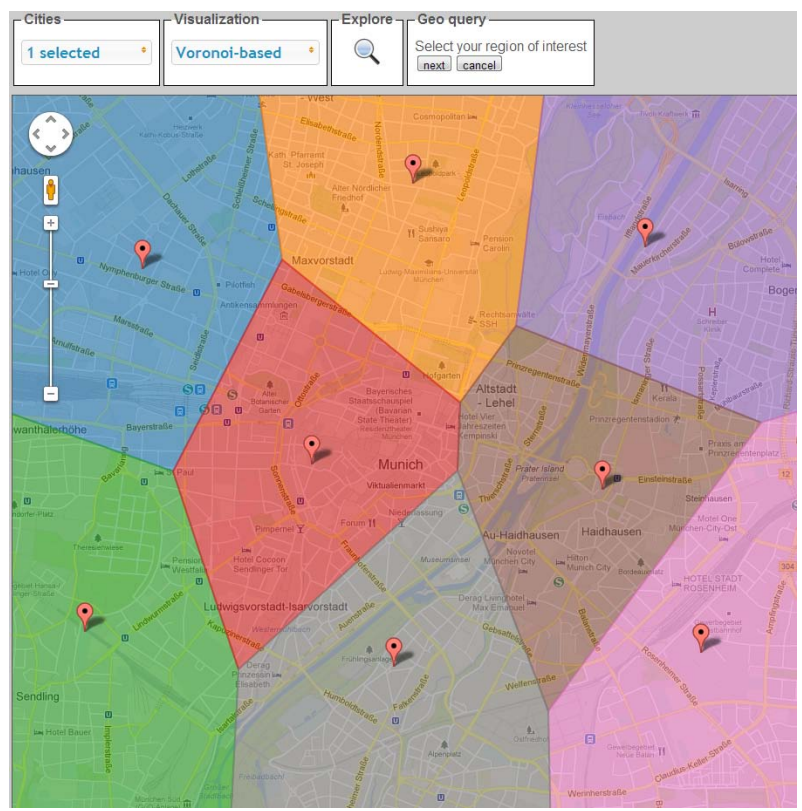

Fig. 6. Voronoi-based heatmap visualization

good overview and assessment on the distribution of geo located facilities across the region and cities, e.g., to identify the regions with better shopping facilities in the city.

Relevance Based Aggregation. Sometimes users need to rank the regions with respect to their criteria of interest, e.g., to find the best place to live or to open a business. We therefore employed a basic multi-criteria aggregation [21] to provide a spatially-related aggregated view on entity distribution with regard to multiple categories. Figure 4 shows the aggregated ranked version of the grid-based visualization. Here each grid cell represents the overall relevance with respect to user selected categories. The relevance score is computed based on the quantity and balanced distribution of geo-entities which belongs to the criteria of interests. The relevance of each grid cell $G$ is based upon user selected category allocation $\left(C_{1}, C_{2}, . ., C_{n}\right)$, where each category $C_{i}$ has an importance based on the geo-entities which belong to category $i$. The relevance of a particular cell $G$ is computed with the combined distribution of categories selected by user. We used a color scheme of six different green tones which differed in their transparency. While light colors represented low relevance, dark colors were used in order to indicate high relevance. The color scheme was chosen with the support of ColorBrewer 5; an online color scheme recommendation tool based on the work of Brewer and Harrower [8].

\footnotetext{
${ }^{5}$ ColorBrewer: http: //www. colorbrewer.org
} 


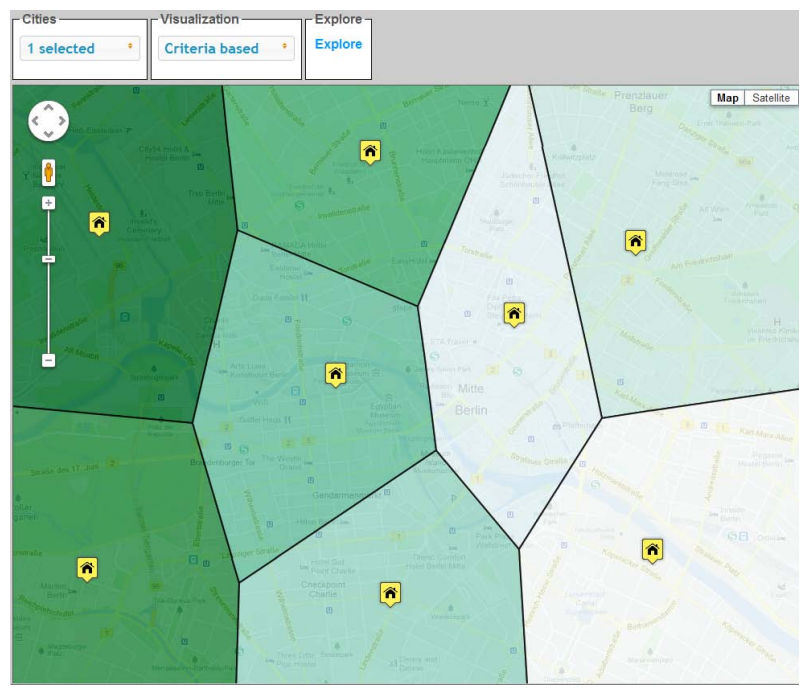

Fig. 7. Aggregated voronoi-based visualization

For the end-user interaction with grid and categories, we show the relevance of a grid with respect to selected categories using a pie chart (Figure 5). The pie charts provide the detailed information on categorical distribution of grid cells when the users click on a particular grid cell with mouse cursor.

\subsection{Voronoi-Based Heatmap Visualization for User-Constrained Spatial Exploration}

The aforementioned grid-based visualization divides the spatial regions in automated fix sized spaces, i.e. the end-user does not have the possibility of specifying personal location preferences as input. The user study also indicated the need of users in preselecting the desired locations and then compares them with respect to their criteria of interest. Figure 6 shows our transformation of heatmap visualization to a voronoi-based division to fulfill such requirement. Voronoi diagram [4] is a way of dividing space into a number of regions. A set of points (seeds) is specified beforehand and for each seed there will be a corresponding region consisting of all points closer to that seed than to any other. The regions are called voronoi cells. The color of each voronoi cell represents the category of largest allocation of geo-entities in the cell area.

Similar to the aggregated view of relevance in grid-based visualization, Figure 7 shows the ranked voronoi-based divisions. Here the relevance of a particular voronoi cell is computed not only based on the distribution of geo-entities; it's additionally the accumulation of root distance of the geo-entities from the selected home location. Figure 8 shows the distribution of geo-entities across the selected locations of city Leipzig with respect to categories: shopping, education and medical facilities. We could see that the most relevant region (voronoi cell 


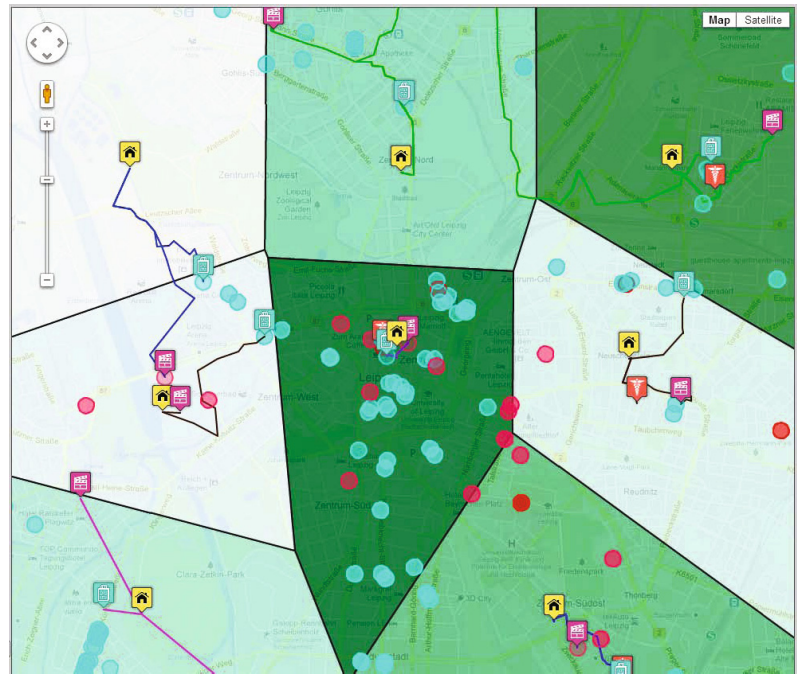

Fig. 8. Aggregated voronoi-based visualization with distribution of geo-entities

with the darkest green shade) has the better distribution of geo-entities and the corresponding home location is closely connected to the geo-entities of interest.

\section{Conclusion and Future Work}

Exploration of multiple criteria for location-based decision making is a common scenario of our daily life, but the current means of local search interface does not offer adequate support for such task. This was also evident from our study when the participant reported on the incapability of Google maps and given GIR interface to accomplish the task of exploring city with multiple criteria. In this paper we argue the need of interactive visual interfaces for a geographic information retrieval system, so the end-user can easily explore and interact with the spatial database. We presented an extension of current interfaces (with multiple placing of markers) to perform multi-criteria search. But the huge amount of markers presented in the marker condition carry the information overload and put high mental demand to perform the task of exploration and comparison of spatial regions. We proposed the use of heatmap visualization through grid and voronoi-based conditions to facilitate the knowledge based exploration of geospatial databases with less information overload. The proposed visualizations has been used in many of visualization research prototypes, but the acceptability of such heatmap based visualization has not been studied in the context of geographic information retrieval for lay users.

Most of the users who participated in the preliminary study were constantly involved in the design and assessment of the interfaces described in this work, still the comparative study of proposed interfaces is essential. In future we plan the 
extensive qualitative and quantitative evaluation of these interfaces, to conclude the acceptability of these visualizations with regard to user-centered aspects such as exploration ability, information overload and cognitive demand. The methodology discussed by Longo et al. 1514 would be a guideline to evaluate the proposed interfaces, and to judge the human mental workload in web-design imposed by different interfaces. We would also like to investigate more advanced interaction methods to enhance the usability of proposed visualizations.

Acknowledgments. The authors are grateful to the DFG SPP priority program 6 which funds the project UrbanExplorer.

\section{References}

1. Ahlers, D., Boll, S.: Location-based Web search. In: Scharl, A., Tochtermann, K. (eds.) The Geospatial Web. Springer (2007)

2. Ahlers, D., Boll, S.: Retrieving Address-based Locations from the Web. In: GIR 2008: Proceedings of the 5th International Workshop on Geographic Information Retrieval. ACM, New York (2008)

3. Ahlers, D., Boll, S.: Adaptive Geospatially Focused Crawling. In: CIKM 2009. ACM (2009)

4. Aurenhammer, F.: Voronoi diagrams: a survey of a fundamental geometric data structure. ACM Comput. Surv. 23(3), 345-405 (1991)

5. Dykes, J., MacEachren, A.M., Kraak, M.-J.: Exploring Geovisualization. International Cartographic Association Series. Elsevier (2005)

6. Fisher, D.: Hotmap: Looking at geographic attention. IEEE Transactions on Visualization and Computer Graphics 13(6), 1184-1191 (2007)

7. Greene, R., Devillers, R., Luther, J.E., Eddy, B.G.: GIS-based multiple-criteria decision analysis. Geography Compass 5(6) (2011)

8. Harrower, M.A., Brewer, C.A.: Colorbrewer.org: An online tool for selecting color schemes for maps. The Cartographic Journal 40(1) (2003)

9. Holzinger, A.: Usability engineering methods for software developers. Communications of the ACM 48(1), 71-74 (2005)

10. Keim, D.A., Kohlhammer, J., Ellis, G., Mansmann, F.: Mastering The Information Age - Solving Problems with Visual Analytics. Eurographics (2010)

11. Kumar, C.: Relevance and ranking in geographic information retrieval. In: Proceedings of the Fourth BCS-IRSG Conference on Future Directions in Information Access, pp. 2-7. British Computer Society (2011)

12. Lam, H., Bertini, E., Isenberg, P., Plaisant, C., Carpendale, S.: Empirical studies in information visualization: Seven scenarios. IEEE Transactions on Visualization and Computer Graphics 18, 1520-1536 (2012)

13. Lloyd, D.: Evaluating human-centered approaches for geovisualization. $\mathrm{PhD}$ thesis, City University London (September 2009)

14. Longo, L., Kane, B.: A novel methodology for evaluating user interfaces in health care. In: 2011 24th International Symposium on Computer-Based Medical Systems (CBMS), pp. 1-6. IEEE (2011)

6 http://www.visualanalytics.de 
15. Longo, L., Rusconi, F., Noce, L., Barrett, S.: The importance of human mental workload in web design. In: WEBIST, pp. 403-409 (2012)

16. MacEachren, A.M., DiBiase, D.: Animated maps of aggregate data: Conceptual and practical problems. CaGIS 18(4) (1991)

17. Maguire, M.: Methods to support human-centred design. Int. J. Hum.-Comput. Stud. 55(4), 587-634 (2001)

18. Makropoulos, C., Butler, D.: Spatial ordered weighted averaging: incorporating spatially variable attitude towards risk in spatial multi-criteria decision-making. Environmental Modelling \& Software 21(1), 69-84 (2006)

19. Moere, A.V., Tomitsch, M., Wimmer, C., Christoph, B., Grechenig, T.: Evaluating the effect of style in information visualizations. IEEE Transactions on Visualization and Computer Graphics 18, 2739-2748 (2012)

20. Nivala, A.-M., Sarjakoski, L.T., Sarjakoski, T.: User-centred design and development of a mobile map service. In: Hauska, H., Tveite, H. (eds.) Scandinavian Research Conference on Geographical Information Science, Stockholm, Sweden, June 13-15. Proceedings of the ScanGIS, pp. 109-123. ScanGIS' 2005 (2005)

21. Rinner, C., Heppleston, A.: The spatial dimensions of multi-criteria evaluation - case study of a home buyer's spatial decision support system. In: Raubal, M., Miller, H.J., Frank, A.U., Goodchild, M.F. (eds.) GIScience 2006. LNCS, vol. 4197, pp. 338-352. Springer, Heidelberg (2006)

22. Rinner, C., Raubal, M.: Personalized multi-criteria decision strategies in locationbased decision support. JGIS 10 (2004) 